\title{
Global Transversality, Resonance and Chaotic Dynamics
}


This page intentionally left blank 


\title{
Global Transversality, Resonance and Chaotic Dynamics
}

\author{
Albert C J Luo \\ Southern Illinois University Edwardsville, USA
}


Published by

World Scientific Publishing Co. Pte. Ltd.

5 Toh Tuck Link, Singapore 596224

USA office: 27 Warren Street, Suite 401-402, Hackensack, NJ 07601

UK office: 57 Shelton Street, Covent Garden, London WC2H $9 \mathrm{HE}$

\section{British Library Cataloguing-in-Publication Data}

A catalogue record for this book is available from the British Library.

\section{GLOBAL TRANSVERSALITY, RESONANCE AND CHAOTIC DYNAMICS}

Copyright $(2) 2008$ by World Scientific Publishing Co. Pte. Ltd.

All rights reserved. This book, or parts thereof, may not be reproduced in any form or by any means, electronic or mechanical, including photocopying, recording or any information storage and retrieval system now known or to be invented, without written permission from the Publisher.

For photocopying of material in this volume, please pay a copying fee through the Copyright Clearance Center, Inc., 222 Rosewood Drive, Danvers, MA 01923, USA. In this case permission to photocopy is not required from the publisher.

ISBN-13 978-981-277-111-7

ISBN-10 981-277-111-5 


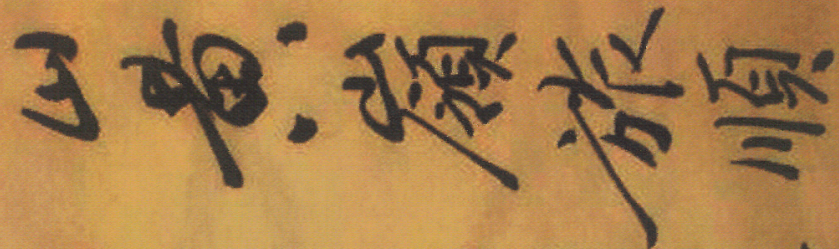

$$
\begin{aligned}
& \text { 3it } \ldots M \text {. }
\end{aligned}
$$

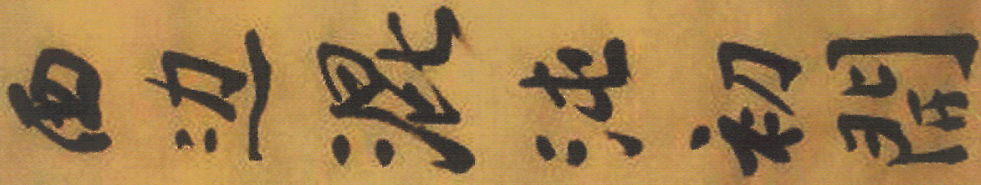

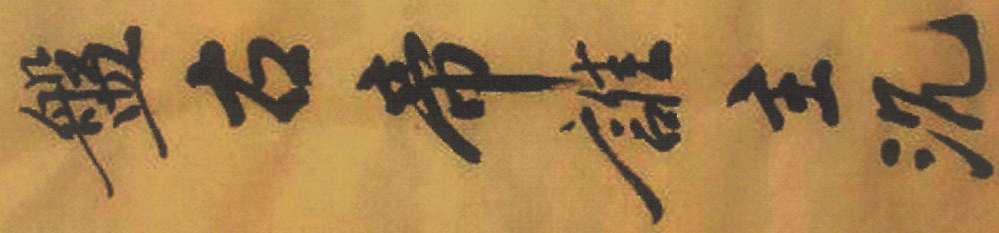

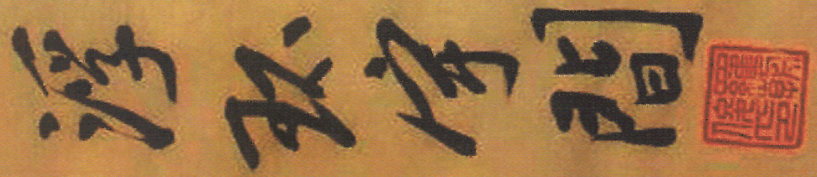

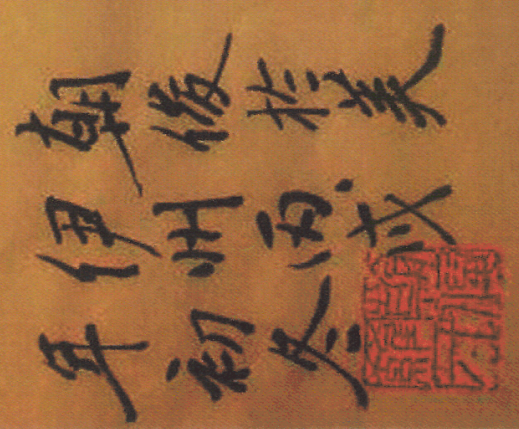


This page intentionally left blank 


\section{Preface}

The modern theory of dynamical systems originates from the Poincarè's qualitative analysis, which focuses on the complexity and stability of motions in such dynamical systems. In 1892, Poincare discovered that the motion of nonlinear coupled oscillators is sensitive to the initial condition and qualitatively presented that the inherent characteristics of the motion in vicinity of unstable fixed points of nonlinear oscillation systems may be stochastic under regular applied forces. This is because the separatrix exists in nonlinear dynamical systems. Such a separatrix connected with hyperbolic points is generic, which is called the generic separatrix. However, another kind of separatrix is generated by the nonlinear resonance between the system and periodic forcing or between the two oscillation interactions. Such a separatrix is called the resonant separatrix. The natural frequency of the separatrix connected with hyperbolic points is zero and the natural frequencies of motion on both sides of the separatrix are different, which makes such a stochastic motion exists in the neighborhood of separatrix. This book will discuss the global transversality of a flow to the separatrix from a domain to another domain in order to understand the mechanism for the onset, growth and destruction of chaos in vicinity of separatrix. The author would like to present a different point of view in order to look into a fundamental theory on global transversality, resonance and chaotic dynamics in nonlinear dynamic systems. The ideas presented in this book are less formal and rigorous in an informal and lively manner. The author hopes the initial ideas may give some inspirations in the field of nonlinear dynamics.

To measure the complex behaviors in a nonlinear dynamical system, the corresponding well-behaved dynamical system is employed. For doing so, the differential geometrical relations of two flows in the two nonlinear dynamical systems are presented. Based on such differential geometric relations, this book presents a theory of global transversality, resonance and chaos in $n$-dimensional nonlinear dynamics. The history and recent development of nonlinear dynamics is briefly discussed first, and then the global transversality of a flow to the separatrix is investigated to determine motion complexity in $n$-dimensional dynamical systems. The resonant mechanism of chaos in $n$-dimensional dynamical systems is discussed in general. Further, the resonant theory of the stochastic layer in 2-dimensional dynamical systems is presented. In addition, the stochasticity of the resonant separatrix layers for $2 n$-dimensional, nonlinear Hamiltonian 
systems is also presented, and nonlinear dynamics on a (2n-1)-dimensional equienergy surface is briefly discussed. For dissipative, nonlinear dynamical systems, the stability and grazing bifurcation are addressed. The global dynamics of 2-dimensional dissipative dynamical systems is presented. Finally, the switchability of a flow from a domain to its adjacent domain in discontinuous dynamical systems is discussed. The objective of this book is to throw out some original ideas on global transversality, resonant dynamics and chaos in nonlinear dynamics. The author believes that some ideas may not be very mature and some typos may exist in the book. The author sincerely hopes that readers can forgive such unavoidable errors here and where in the book. The author really appreciates readers for providing suggestions and comments to improve the theory presented in this book.

This book is dedicated to people who are challenging difficult and unsolved problems in natural science. Finally, I also dedicate this book to my wife (Sherry $X$. Huang) for support and to my lovely children (Yanyi Luo, Robin Ruo-Bing Luo, and Robert Zong-Yuan Luo) for their happiness to stimulate my inspiration.

Albert C.J. Luo

Edwardsville,

Illinois 


\section{Contents}

Preface vii

Chapter 1. Introduction 1

1.1. A brief history of dynamics 2

1.2. Nonlinear Hamiltonian systems 5

1.2.1. Separatrix splitting $\quad 6$

1.2.2. Standard and whisker maps 9

1.2.3. Chirikov resonance overlap criterion $\quad 12$

1.2.4. Renormalization group technique 13

1.3. Dissipative nonlinear systems 15

1.4. Book layout 18

Chapter 2. Differential Geometry of Flows 23

2.1. Normal distance and $G$-functions 23

2.2. Non-contact flows 31

2.3. Contact flows 47

2.4. Concluding remarks 61

Chapter 3. Global Transversality in Continuous Dynamical Systems 63

3.1. Nonlinear dynamical systems $\quad 63$

3.2. Local and global flows $\quad 66$

3.3. Global transversality 71

3.4. Global tangency $\quad 80$

3.5. Perturbed Hamiltonian systems $\quad 88$

3.6. Two-dimensional Hamiltonian systems $\quad 92$

3.7. A damped Duffing oscillator 95

3.7.1. Transversal and tangential conditions 97

3.7.2. Global transversal and tangential flows 101

3.8. Global transversality to a generalized separatrix 104

Chapter 4. Chaotic Layer Dynamics 113

4.1. Chaotic domains in phase space 113

4.1.1. Maximum and minimum first integral surfaces $\quad 114$

4.1.2. First integral quantity intervals 119 
4.1.3. Chaotic motion bands

4.2. First integral quantity increments 127

4.3. Resonance mechanism of chaotic layers $\quad 140$

4.4. Energy increments in perturbed Hamiltonian systems 148

Chapter 5. Two-Dimensional Stochastic Layers 153

5.1. Geometric description in phase space 153

5.2. Approximate predictions 158

5.2.1. An incremental energy method 158

5.2.2. An accurate standard mapping technique 163

5.2.3. Energy spectrums and layer width 164

5.3. Stochastic layer in a Duffing oscillator $\quad 167$

$\begin{array}{ll}\text { 5.3.1. Incremental energy technique } & 170\end{array}$

$\begin{array}{ll}\text { 5.3.2. Accurate standard-mapping approach } & 171\end{array}$

5.3.3. Approximate standard-mapping approach $\quad 173$

5.3.4. Energy spectrum and layer width $\quad 175$

5.3.5. Analytical predictions comparison 178

5.3.6. Illustrations of stochastic layers 186

5.4. Conclusions and discussions 193

Chapter 6. Stochasticity in Resonant Separatrix Layers 197

6.1. Two-dimensional resonant separatrix layers 197

6.1.1. Layer dynamics 199

6.1.2. Approximate predictions 210

6.1.2a. Onset conditions 211

6.1.2b. Vanishing conditions 214

6.1.2c. Energy increment spectrum 215

6.2. $2 n$-dimensional resonant separatrix layers $\quad 216$

6.2.1. Internal resonant layer dynamics 218

6.2.2. External resonant layer dynamics 220

6.2.3. Approximate criteria 221

6.3. Resonant layers in a Duffing oscillator 222

6.3.1. Resonant layer dynamics 223

6.3.2. Analytical conditions 227

6.3.3. Numerical predictions $\quad 230$

6.3.4. Illustrations of resonant layers 234

6.4. Resonant layers in a parametric pendulum 237

6.4.1. Librational resonant layer 238

6.4.2. Rotational resonant layer 243 
Chapter 7. Nonlinear Dynamics on an Equi-energy Surface

7.1. Hamiltonian systems

7.2. Nonlinear resonance 255

7.3. Energy spectrum 258

7.4. Chaotic motions on an equi-energy surface 261

7.4.1. Resonance and averaging of Hamiltonians 263

7.4.2. Energy exchanges and criterion 265

7.4.3. Energy spectrum computation 268

7.4.4. Numerical simulations 278

7.5. Conclusions 288

Chapter 8. Stability and Grazing in Dissipative Systems 289

8.1. Equilibrium stability 289

8.1.1. System dissipativeness and expansiveness 290

8.1.2. Stability definitions 293

8.1.3. Stability conditions 295

8.2. Periodic flow stability 302

8.2.1. Stability conditions 303

8.2.2. Limit cycles 311

8.3. Local grazing bifurcation $\quad 314$

8.3.1. Local grazing $\quad 315$

8.3.2. Grazing mapping $\quad 316$

8.3.3. Grazing determination 321

8.4. Global grazing bifurcation $\quad 324$

8.4.1. Global grazing and singular sets 325

8.4.2. Global grazing conditions 326

8.4.3. Global strange attractor fragmentation 337

Chapter 9. Global Dynamics in Two-dimensional Dynamical Systems 341

9.1. Tangency and transversality 341

9.2. Energy increment and Melnikov function 343

9.3. Mapping structures $\quad 348$

9.4. Bifurcation scenario $\quad 356$

9.5. Numerical illustrations $\quad 362$

9.5.1. Periodic flows 362

9.5.2. Grazing periodic flows $\quad 369$ 
9.5.3. Poincarè mapping sections of chaos

9.5.4. Global transversality of chaos

Chapter 10. Flow Switchability in Discontinuous Dynamical Systems 